\title{
Research and Thinking on the Development of Hospital Informatization under the New Situation
}

\author{
Liangwei Yao ${ }^{1}$, Qian Zhang ${ }^{2 *}$, Xuehui $\mathrm{Li}^{1}$ and $\mathrm{Li} \mathrm{Xu}^{1}$ \\ ${ }^{1}$ School of Information Science and Engineering, Zaozhuang University, Zaozhuang, Shandong, 277100, China \\ ${ }^{2}$ Information Department, People's Hospital of Xuecheng District, Zaozhuang, Shandong, 277100, China
}

\begin{abstract}
With the vigorous advancement of China's medical reform and the continuous improvement of medical services, hospitals are increasingly demanding and relying on informatization. It can be said that informatization covers all aspects of hospital business and provide comprehensive, efficient, convenient and automated management and services for the normal operation of the hospital. At present, hospitals have gradually increased their investment in informatization, and the upgrading and transformation of informatization has also been continuously enhanced. However, there are still a large number of problems in the application of hospital informatization management. It is necessary for hospital informatization managers and executors to face relevant problems, understand the tasks and goals of the next stage of hospital informatization development. This paper mainly expounds the development status of hospital information system, lists the common problems in the application of hospital informatization, puts forward reasonable and effective informatization construction methods and strategies, and points out that the construction of integrated platform hospital data warehouse and health care cloud platform is the future development direction of hospital informatization.
\end{abstract}

\section{Introduction}

In recent years, in order to adapt to various medical policies and new transformation needs, some old hospital systems are facing elimination or upgrading[1], various medical application software systems have emerged as the times require, and each hospital's system has its own merits, thus forming its own characteristics of the application of information technology. This article has carried out a detailed inventory and summary of the development of hospital informatization, analyzed the problems and challenges faced in the informatization process, researched and put forward constructive methods and strategies, and pointed out the future development direction of hospital informatization.

\section{The development status of hospital information system}

China's medical informatization was founded in the 1980s. According to the development of medical informatization, the development process of medical informatization can be roughly divided into four stages: the initial stage of hospital information system (HIS), the stage of hospital clinical system with HIS as the core, and the stage of clinical information system(CIS)[2] based on electronic medical record(EMR)construction stage and smart hospital construction stage.

\subsection{The first stage}

Very few medical institutions are in the initial stage of using Hospital Information System (HIS). The initial HIS system includes outpatient and emergency registration fees, outpatient doctor workstations, hospitalization management, drug store management, pharmacy management, etc. The system is only used for financial and internal hospital management purposes. The information system in this period is called management information system. The framework is generally $\mathrm{C} / \mathrm{S}$ architecture. The hospital system data at this stage of development are independent of each other, forming islands of information.

\subsection{The second stage}

Some medical institutions are in the hospital clinical system stage with his system as the core. His system in this stage is a comprehensive management system which mainly deals with the flow of people, finances, logistics and other information of the hospital and its subordinate departments. It is oriented to the internal management of the hospital. It takes the managers at all levels of the hospital as the service object, and takes the repetitive things processing as the basic management unit, so as to 
realize the hospital information management and improve the hospital management efficiency. The system includes laboratory information management system (LIS), pictrue archiving and communication system (PACS), electronic medical record (EMR), medical insurance settlement, financial management, performance management, etc, cover all functional departments, and multiple systems have realized partial information sharing. The information management system centered on HIS is shown in Figure 1.

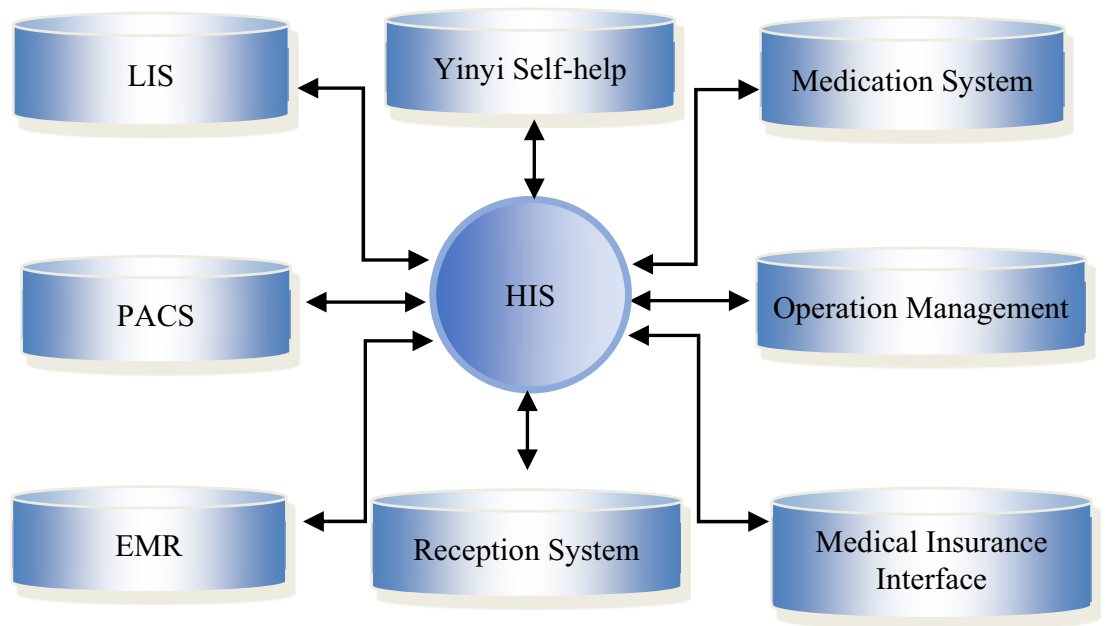

Figure 1. Diagram of an information management system with HIS as the core

\subsection{The third stage}

Most medical institutions implement clinical information system (CIS) on the basis of electronic medical records ${ }^{[2]}$. The system is a management system which mainly deals with clinical information, and medical process processing based on medical knowledge is the basic management unit. It realizes the purpose of improving medical quality for clinical medical management, which is based on patients, medical staff as service object and facing clinical medical management. The new system includes: secondary database management, operation anesthesia management, blood transfusion system, rational medication, intensive care system, mobile medical treatment, quality control system and other systems. The information sharing degree is higher than that of the second stage, and the data interaction of information system is greatly enhanced. The clinical information system with electronic medical records as the core is shown in Figure 2.

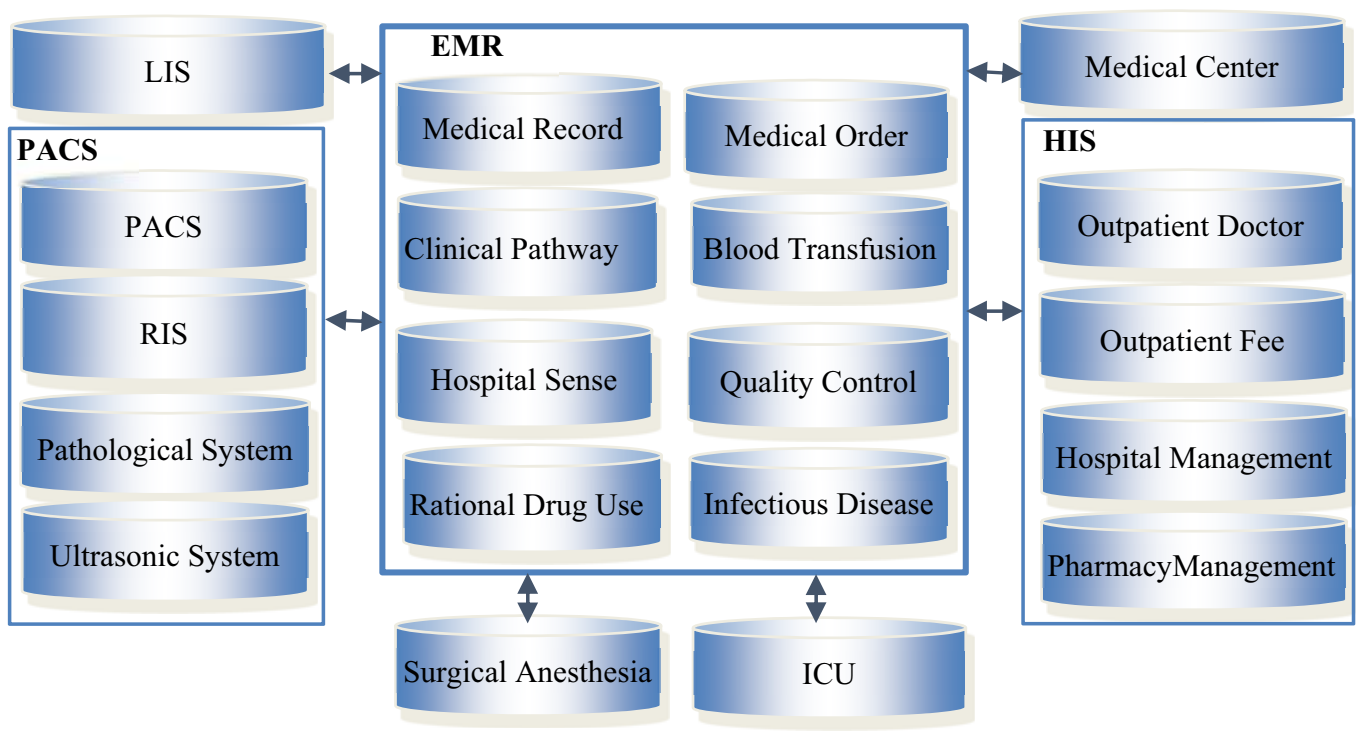

Figure 2. Diagram of clinical information system centered on electronic medical records

\subsection{The fourth stage}

The construction stage of a smart hospital[3]. Smart hospital refers to the establishment of a complete medical information management and service system based on high-tech technologies such as the Internet of things and cloud computing in diagnosis, treatment, rehabilitation, payment, and health management.To achieve functions such as medical information interconnection, sharing and collaboration, clinical innovation, and diagnostic science. In 2019, the National Health Commission proposed a three-in-one smart hospital construction idea, and clarified the path of hospital informatization[4]. These three aspects include smart medical care, smart service, and 
smart management. At present, some tertiary hospitals have established integrated platforms and data centers to realize the standardization and interconnection of business interaction data, and create an integrated architecture system of smart medical care, smart services, and smart management under the framework of information security assurance. The architecture system diagram of the smart hospital platform is shown in Figure 3.

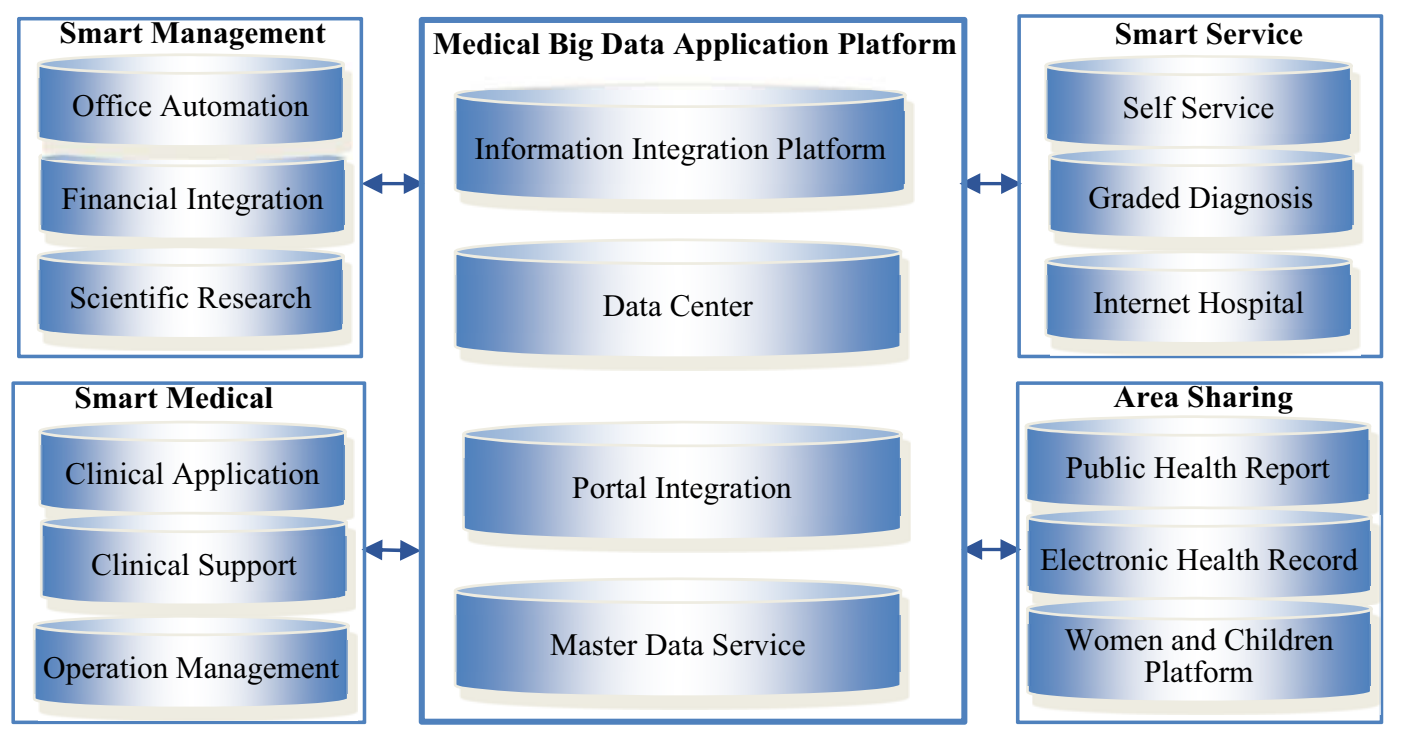

Figure 3. Smart hospital platform architecture system

Among them, smart service takes patients as the service object, allowing patients to experience a more convenient and faster way to seek medical treatment[5]. The use of self-service kiosks, mobile phone official accounts, APP and other equipment, including mobile phone settlement, appointment registration, appointment diagnosis and treatment, inspection result inquiry, and medical record copying and mailing to home are all smart services for patients. Especially during the new crown pneumonia epidemic, smart services have significantly improved the results of medical services and played an active role in responding to the epidemic and meeting the needs of the people for medical treatment. The following is the use of smart services such as self-service and appointment registration in a hospital from April 2020 to April 2021, as shown in Figure 4 and Figure 5.

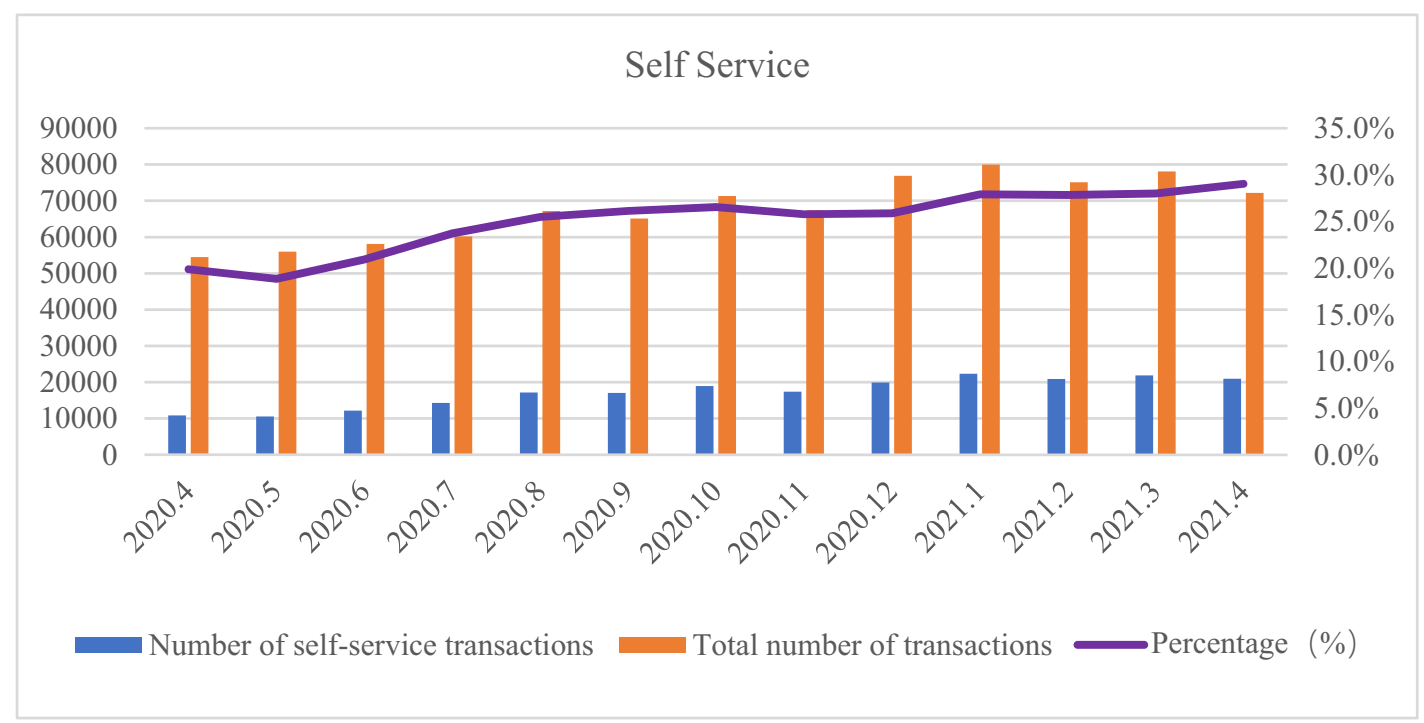

Figure 4. Self-service usage of a hospital in Zaozhuang 


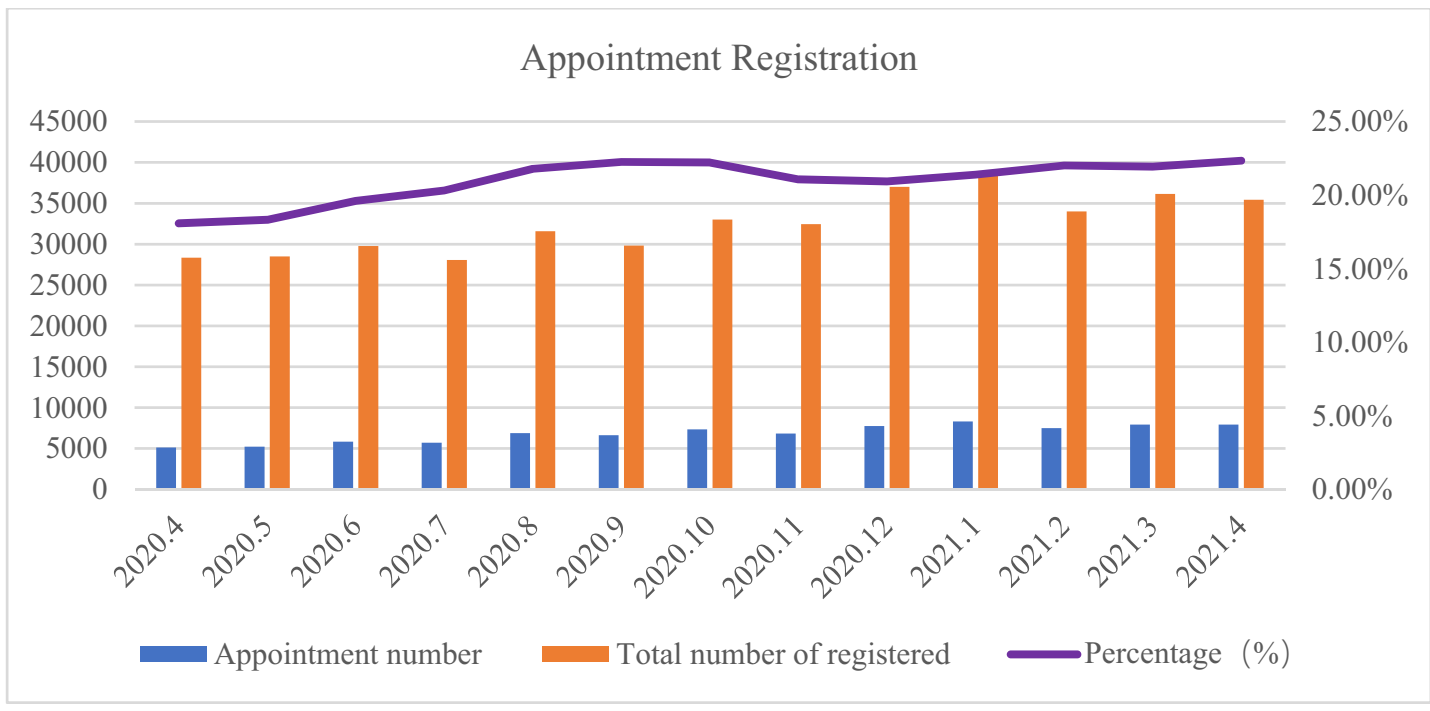

Figure 5. The use of appointment registration in a hospital in Zaozhuang

From the above charts, it can be seen that the proportion of self-service transactions and the proportion of appointment registration is gradually showing a steady upward trend. In April 2021, the proportion of self-service transactions is as high as $29 \%$, and the proportion of appointment registration remains between $20 \%-25 \%$. Hospitals are vigorously promote the use of self-service equipment and appointment registration, which not only saves a lot of manpower and resources for the hospital, but also makes it easier and faster for patients to seek medical treatment.

\section{Common problems in the application of hospital information}

In the process of hospital informatization construction, there are problems such as lack of overall planning, implementation of the concept of building a society, low attention, insufficient capital investment, uneven construction standards, insufficient resource integration and utilization, etc., which require hospital managers to have a forward-looking vision and Open thinking, reexamine and plan informatization construction with the concept of development. At the same time, the construction and application of informatization requires the coordination and cooperation of the whole hospital[67]. The common problems in the application of hospital informatization are summarized as follows:

\subsection{The overall planning is lacking and the concept of building a society is backward.}

Starting in 2019, the smart service classification review work organized by the National Health Commission's Medical Administration and Hospital Management Bureau mainly involves hospital smart services[8-9]. The participation of primary medical institutions is very low, and there is a lack of overall planning and policy standards. The concept of building a society is mostly "to meet the needs of medical staff, and there is no unified demand control". After the needs of one stage are met, the next step of the informationization plan is not continued, and the overall direction of the informationization of the hospital management is not grasped from the top level of the hospital.

\subsection{The degree of attention is not high, and the capital investment is insufficient.}

The importance of medical institutions on informatization needs to be strengthened. The management of a small number of medical institutions has only a superficial understanding of informatization. They believe that informatization is to buy technology and systems based on business needs, and is eager to see actual application effects in the short term. At the same time, because the medical income brought by the capital investment in the information construction is not obvious, most medical institutions lack stable capital investment in the information construction, so they cannot meet the information construction needs of the medical institutions, resulting in the slow progress of the information construction.

\subsection{Insufficient staffing and unclear department responsibilities.}

The daily implementation and maintenance of the information system of medical institutions is intensive, and the information management staff is relatively insufficient. There are fewer compound talents who understand both medical knowledge and information technology. Once the system has problems, the information management staff cannot solve the problem in time and need to contact the software. The company or technicians solve the problem, and there are technical dependence and management defects. In addition, the clinical department and the information department have problems such as poor communication, mutual complaints, and disconnected collaboration. At the same time, the information operation ability of medical staff needs to be improved, which greatly hinders the rapid development of hospital informatization. 


\subsection{System construction is scattered, and it is difficult to find problems.}

Each information system put into use can only effectively solve the targeted problems, and it is difficult to integrate into a whole, so as to disperse into independent subsystems. Because of the large number of subsystems, sometimes doctors, nurses, pharmacists and technicians need to open 3-6 different systems at the same time to complete a whole set of operation, which is cumbersome and greatly reduces the work efficiency. Diversified subsystems need to be connected by various data interfaces. If a subsystem has a problem, it is necessary to check all related systems. The speed and difficulty of finding and solving the problem increase, which brings great trouble to information management and medical work, and leads to the poor overall efficiency of informatization.

\subsection{The degree of attention is not high, and the capital investment is insufficient.}

With the continuous advancement of informatization, information and network security has become more and more important to everyone. However, due to the weak awareness of information security in some medical institutions, the lack of professional and technical standards, and the imperfect network security protection system, information leakage, Unexpected situations such as information tampering, virus infection, security breaches, hacking attacks, and even paralysis of the business system occur from time to time, hindering the normal development of medical work, undermining the security of the hospital's information system and the confidentiality of information, and affecting stability and security of medical informatization.

\section{Methods and strategies of hospital informatization construction}

The construction of hospital informatization is an important pillar of the medical and health information system. The higher the degree of informatization, the better the medical work of the majority of medical workers and the improvement of work efficiency. At the same time, it can also provide patients with a fast and satisfactory medical experience. Aiming at the problems in the process of informatization construction, this article proposes relevant solutions and strategies as follows:

\subsection{Improve the overall plan and change the construction concept}

The overall structure is the soul and foundation of the entire hospital's information system construction. The hospital should follow the National Health Commission's overall requirements for hospital informatization construction standards and norms, and use the characteristics of hospital management, business requirements and system applications to formulate a hospital The long-term development plan and strategic goal of informatization construction. At the same time, continue to learn from well-developed high-level domestic and foreign hospitals, and learn from their efficient information management model and application technology[10]. Change the traditional, backward and passive informatization construction thinking, build an informatization system with the service concept of "all patients as the center", and provide safe, convenient and efficient informatization services.

\subsection{Improve ideological position and increase capital investment}

Hospital decision-makers and managers should attach importance to hospital informatization construction[11], stand on the height of the development of the times, take regional health and medical informatization construction as the goal, and provide scientific direction guidance for the development and practice of informatization. Sound and stable capital investment is the driving force for the development of hospital informatization. The hospital should analyze and judge the informatization construction every year, formulate construction plans and capital budget plans, and make dynamic adjustments according to the actual operation and financial situation of the hospital, and appropriately increase the needs for informatization. The investment in software and hardware of the hospital has escorted the rapid development of hospital information development.

\subsection{Strengthen team building and clarify department responsibilities}

With the increasing workload and work intensity of information management personnel, medical institutions must improve the employment system, focus on the selection and excavation of information technology talents, introduce more compound talents required for high-level information management, and carry out information management on a regular basis Professional knowledge training of personnel continuously improves the professional technology and management level of information management personnel. At the same time, the information department should organize more medical staff to participate in system application and basic computer skills training to continuously improve the information operation ability of the entire hospital. In addition, medical institutions must clarify department responsibilities, clinical departments and information departments must strengthen communication and coordination, and participate in the planning and construction of information systems in an all-round way.

\subsection{Reasonably integrate subsystems and give full play to informatization}

Hospital informatization construction is no longer the sum of the "project construction" of various systems. It is necessary for the hospital to closely contact the actual business, combine the hospital's own development goals, and put forward corresponding construction plans in 
accordance with the rules, models and methods of hospital informatization construction. Promote the construction of hospital information integration platform for specific topics and needs, and gradually realize the simplification, standardization, modularization and integration of related business systems within the hospital and within the region, so as to ensure lower cost and higher flexibility sustainable development of health information construction[12].

\subsection{Strengthen information security precautions and attach importance to network security protection}

Network information security is the bottom line of hospital development. In order to effectively prevent the leakage of important information such as patient privacy and hospital business information, it is necessary to establish a comprehensive protection system to protect network information security, passing identity authentication, virtual private network, access network permissions, and data encryption and other technologies ensure that information is not leaked. Information management personnel also visit the central computer room of the hospital every day to check network security, data backup, and hardware equipment security. Hospitals should pay attention to network information security training, strengthen the awareness of network information security, introduce advanced experience and technology at home and abroad, and continuously optimize their own network security system architecture.

\section{The future development direction of hospital informatization}

\subsection{The integrated platform hospital data warehouse.}

The integrated platform hospital data warehouse enables the interconnection of different subsystems to form a standardized management system[13]. It is conducive to the integration and sharing of data, greatly enhances the interoperability of information system data, promotes good communication between the hospital and the patient, facilitates the medical staff to fully grasp the patient's condition, allows the patient to quickly receive precise treatment, and realizes the optimal management of medical services And closed-loop control of the diagnosis and treatment service process. At the same time, effective supervision and scientific decision-making of medical institutions can be realized, and the core competitiveness of various functional departments and clinical departments in data utilization and medical institution services can be improved[14].

\subsection{The health care cloud platform.}

The health care cloud platform is an upgrade and expansion of the hospital information platform based on cloud computing technology architecture[15]. It adopts a flexible architecture and big data solutions. It is internally interconnected with the hospital information system through the hospital service bus, and the external and regional population is healthy. The information platform is interoperable to form a medical data center within the hospital and the medical consortium/medical community, which gathers service functions and data such as operation management, clinical services, medical documents, and health files to carry out medical treatment, teaching, scientific research, health management, and health management for the hospital. Auxiliary decision support provides support.

\section{Conclusion}

This paper mainly expounds the development status of hospital information system, puts forward reasonable and effective solutions to the common problems encountered in the process of hospital informatization, and takes the construction of integrated platform hospital data warehouse and health care cloud platform as the focus and direction of hospital informatization research in the future. At present, the development of hospital informatization is still facing many bottlenecks, but with the development and popularization of information technologies such as Internet + , smart hospitals, cloud computing, and big data, a new situation in the development of hospital informatization will surely be created. As long as the hospital optimizes the hospital management model as a whole, pays attention to informatization construction and planning, scientific coordination, overall planning, and steady advancement of hospital informatization construction, improving the efficiency of medical services, allowing patients to run less and more information, and at the same time, speed up information system interconnection and sharing, strengthen medical quality supervision and information security protection work, and promote the continuous development of medical and health undertakings.

\section{Acknowledgments}

This work is supported by the Youth Program of the Natural Science Foundation of Shandong Province (NO: ZR2020QF110) and General project of Zaozhuang University(NO: 102061903).

\section{References}

1. Mao, D., Kong, Y.L. (2019) Information Construction and Operation Status in National Hospitals of Grade Two and Grade Three. Modern Hospital Management, 17: 57-60.

2. He, L.,Wang, L.X. (2019) Analysis on the situation and problems of information system construction in hospital. Soft Science of Health, 33: 96-98.

3. Xu, R.R., Zhang, Y., Zhou, B.Y., et al. (2018) Research on the Construction and Application of Information Platform in the Construction of Smart Hospital. Chinese Hospital Managemen, 38: 55-57. 
4. Chen, P., Li, J.H. (2020) Thinking on hospital informatization construction of "Internet plus smart medicine". Chinese Information, 7: 87-88.

5. Luo, J. (2018) Design of smart medical system based on mobile Internet and big data. Electronic Technology \& Software Engineering, 4: 132.

6. Li, X.Y. (2019) Research on the current situation and Development Countermeasures of hospital information construction in China. Medical Information, 32: 1-2.

7. Tan, Z.C. (2019) Problems and Countermeasures in Informatization Construction of Grass-roots Hospitals. China Health Standard Management, 10: 3-5.

8. Xu, T., Cui, W.B., Yu, G.J. (2020) Construction status and development path of smart hospitals in China. Chinese Hospitals, 24: 1-3+10.

9. Wang, Q.J., Shi, W.D., Li, Y., et al. (2020) Study on Service Accessibilit of Smart Hospital under "Internet +" Mode. Journa of Medical informatics, 41: 48-51.

10. Wang, B. (2020) Research on the development of hospital management mode under the new information situation. Electronic Journal of General Stomatology, 7: 20+25.

11. Wei, T.Y. (2020) role of hospital informatization construction in hospital development. China Health Care \& Nutrition, 29: 370.

12. Yang, L. (2020) Research on the Problems and Countermeasures of Public Hospital Information Construction under the Background of New Medical Reform. Qingdao University, Qingdao.

13. Sun, Y.L., Yang, X.J. (2019) Discussion on hospital information management. China Health Industry, 16: 174-176.

14. Huang, Y., Wei, L., Zhang, L., Fei, X.L. (2019) Construction and application of information integration platform of hospital based on big data. China Medical Equipment, 16: 103-105.

15. Wang, P., Zhou, J., Wang, K.X., et al. (2020) A Review on Healthcare Big Data Cloud Platform. China Medical Devices, 35: 161-165+174. 